\title{
Raman and Infrared Spectroscopic Tentative Identification of Organic Traces in Sadiya (LL5) Ordinary Chondrite
}

\author{
Bhaskar J. Saikia ${ }^{*}$, G. Parthasarathy ${ }^{2}$ and Rashmi R. Borah ${ }^{3}$ \\ ${ }^{1}$ Department of Physics, Anandaram Dhekial Phookan College, Nagaon, India \\ ${ }^{2}$ National Geophysical Research Institute (CSIR-NGRI), Hyderabad, India \\ ${ }^{3}$ Department of Physics, Nowgong College, Nagaon, India \\ Email: vaskaradp@gmail.com
}

\begin{abstract}
We report here for the first time the possible presence of organic compounds in Sadiya (LL5) ordinary chondrite using micro-Raman and infrared spectroscopic technique. The micro-Raman spectrum exhibits the diamond and graphite peaks correspondingly at $1331 \mathrm{~cm}^{-1}, 1349 \mathrm{~cm}^{-1}$ and 1588 $-1618 \mathrm{~cm}^{-1}$. The full wave at half maximum value of about $18 \mathrm{~cm}^{-1}$ for Sadiya indicating the nature of disordered phase involved shock metamorphism in the meteorite samples. The diamond and graphite peaks intensity ratio $(\sim 0.53)$ indicates the disordered nature of graphite. The infrared spectrum in the range 2700-3000 $\mathrm{cm}^{-1}$ indicates the presence of $\mathrm{CH}_{3}$ asymmetric stretching, and $\mathrm{CH}_{2}$ symmetric and asymmetric stretching modes due to aliphatic hydrocarbons. This study has strong implications in understanding of the organic compounds in extra-terrestrial materials.
\end{abstract}

Keywords: Sadiya meteorite, Raman, infrared, organic compound.

\section{Introduction}

Meteorites are an important source of extraterrestrial matter, their chemical and physical characteristics, texture and internal structure contribute to our understanding of the birth and early history of our solar system [1]. The primitive meteorites contain abundant amounts (up to $1500 \mathrm{ppm}$ ) of nanodiamonds [2, 3]. The main formation process of the meteoritic nanodiamonds may be either in chemical vapour deposition or in shock origin (e.g., [4]). These nanocrystalline carbon materials (presolar grains) have to be considered amorphous or glassy carbon, and are either inorganic or consist of hydrogenated aromatic macromolecular organic carbon in the Raman spectroscopic literatures (e.g., see [5-8]). Alternatively, the carbon in primitive chondritic meteorites is present as organic material in the form of solvent -soluble and -insoluble components $[9,10]$. Raman spectroscopy is a non-destructive technique that can yield information on the structural order of polyaromatic organic matter when applied at low laser power. Therefore, it is used to investigate insoluble organic matter from a range of chondritic meteorites, and a suite of interplanetary dust particles [11-13]. Raman analysis of insoluble organic matter has been already discussed by various authors in different meteorites [14-17], as well as of interplanetary dust particles [18-20] and returned samples from the Stardust Mission [21, 22]. The organic compounds in the dense interstellar clouds exhibit infrared absorption features at $\sim 2880 \mathrm{~cm}^{-1}$ or $3.4 \mu \mathrm{m}[23,24]$. The existence of similar features (at $3.4 \mu \mathrm{m}$ interstellar band) in Dergaon meteorite by infrared spectroscopy has been discussed by Saikia et al. [10].

This study demonstrates the presence of organic tracers in Sadiya LL5 ordinary chondrite which fell at Natun Balijan village of Sunpura, Sadiya $\left(27^{\circ} 50^{\prime} 09^{\prime \prime} N\right.$; 95 $\left.51^{\circ} 34^{\prime \prime} E\right)$, India, on $5^{\text {th }}$ June 2017 (04:30 pm IST)[25] using Raman and infrared spectroscopic technique. Commonly Organic carbon including PAH are found in carbonaceous meteorite. However the organic carbon in ordinary chondrite is very rare. In our study we present our new results on occurrence of organic carbon in ordinary chondrite.

\section{$2 \quad$ Experimental}

All the sample preparation was performed in ultra-clean conditions. To prevent from the environmental artefact contaminations, such as mud, the sample was carefully checked by optical microscopy. To avoid any surface contamination and the fusion crust we fragmented the sample $(\sim 20 \mathrm{mg})$ and took only pieces 
coming from its interior. The Raman spectra were collected on bulk powdered meteorite samples using an $\mathrm{Ar}$ ion laser laser with a power of $\sim 5 \mathrm{~mW}$, which used an excitation source having wavelength 488 nm coupled with a Jobin-Yvon Horiba LabRam-HR Micro-Raman spectrometer equipped with an Olympus microscope with 10X, 50X and 100X objectives, using the method described elsewhere [26, 27]. The powdered sample was homogenized in spectrophotometric grade $\mathrm{KBr}(1: 20)$ in an agate mortar and was pressed $3 \mathrm{~mm}$ pellets with a hand press. We tried to minimize the grinding time to avoid the deformation of the crystal structure, the ion exchange and the water absorption from atmosphere. The infrared spectra was acquired using Perkin-Elmer system 2000 FTIR spectrophotometer with heliumneon laser as the source reference, at a resolution of $4 \mathrm{~cm}^{-1}$. The spectra were taken in transmission mode in the region $400-4000 \mathrm{~cm}^{-1}$. The room temperature was $29^{\circ} \mathrm{C}$ during the experiment. The $800-$ $1100 \mathrm{~cm}^{-1}(10 \mu \mathrm{m})$ and $800-400 \mathrm{~cm}^{-1}(20 \mu \mathrm{m})$ region of the infrared spectra facilitate an understanding of the relative nature of the $\mathrm{SiO}_{4}$ tetrahedra. Particular interest is directed towards the presence of trace of organic compound in the spectral region around $2800-3000 \mathrm{~cm}^{-1}(3.4 \mu \mathrm{m})$, due to the aliphatic functional $\mathrm{CH}_{2}$ and $\mathrm{CH}_{3}$ groups.

\section{Results and Discussion}

The compositional, mineralogical and Raman spectroscopic analyses of Sadiya meteorite have been discussed by Saikia et al. elsewhere (see [25]). The olivine and pyroxene composition of Sadiya meteorite are determined as: $\mathrm{Fa}_{28.97} ; \mathrm{Fo}_{71.03} ; \mathrm{Fs}_{24.47} ; \mathrm{En}_{74.03}$ and $\mathrm{Wo}_{1.5}$. The Raman spectra exhibit two characteristics peaks of olivine at $820 \mathrm{~cm}^{-1}$ and $850 \mathrm{~cm}^{-1}$ and three characteristic peaks of pyroxene at $335 \mathrm{~cm}^{-1}, 679 \mathrm{~cm}^{-1}$ and $1004 \mathrm{~cm}^{-1}$. The olivine and pyroxene phases have already been discussed by the author in [25], therefore, the emphasis is given to the carbon phases in the Raman spectra of the meteorite.

Figure 1 displays different carbon phases observed in Raman spectra in between 1100-2000 $\mathrm{cm}^{-1}$. In general, the Raman spectra of nanodiamonds reveal two broad bands centred at $1326 \mathrm{~cm}^{-1}$ and $1590 \mathrm{~cm}^{-1}$ [26]. The first-order Raman band $\left(\mathrm{F}_{2 \mathrm{~g}}\right)$ of diamond occurring at $\sim 1332 \mathrm{~cm}^{-1}$ corresponds to carbon $\operatorname{sp} 3$ bonding (the main $\mathrm{C}-\mathrm{C}$ bond vibration in diamond) and the band $\left(\mathrm{E}_{2 \mathrm{~g}}\right)$ at $\sim 1590 \mathrm{~cm}^{-1}$ is assigned to carbon sp2 bonding graphitic structures [28]. The peaks exhibiting in the Raman spectrum (Fig.1) at $1331 \mathrm{~cm}^{-1}, 1349 \mathrm{~cm}^{-1}$ and $1588-1618 \mathrm{~cm}^{-1}$ attributed to diamond and graphite (D and G carbon bands), respectively. The peak intensity is generally used as a signature of crystalline quality. The relative intensities of these two bands reflect the degree of ordering [29]. The peak intensities of these bands are relatively low, which indicate strong background fluorescence. Depending on the degree of disordering and orientation of the graphite grain, a second band around $1350 \mathrm{~cm}^{-1}$ and a shoulder at $1620 \mathrm{~cm}^{-1}$ of lower intensity is present [30], which can be observed in the Raman spectrum of Sadiya meteorite at $1349 \mathrm{~cm}^{-1}$ and $1618 \mathrm{~cm}^{-1}$. The relative intensities of the $1349 \mathrm{~cm}^{-1}$ and $1588 \mathrm{~cm}^{-1}$ peaks reflect the degree of ordering or disordering [29]. The Raman peak position $1618 \mathrm{~cm}^{-1}$ is the evidence of disordered graphite in the Sadiya meteorite (Fig. 1).

The peak appearing at $1331 \mathrm{~cm}^{-1}$ is accepted for diamond, such Raman peaks at $\sim 1318-1333 \mathrm{~cm}^{-1}$ for apparently shock-produced diamond inside graphite nodules from Canyon Diablo were found by Miyamoto [31]. Diamond formation by chemical vapour deposition at low pressures and high temperatures is also possible in stellar atmospheres where the nanodiamonds of chondrites [32] were generated. The artificially produced chemical vapour deposited nanocrystalline diamonds exhibit two significant bands at $1150 \mathrm{~cm}^{-1}$ and $1450 \mathrm{~cm}^{-1}$ [33]. Similar peak at $1455 \mathrm{~cm}^{-1}$ is observed in Sadiya spectra. Therefore, it may be believed that the formation of nanodiamonds in Sadiya is similar to this process (chemical vapour deposition).

In general, FWHM 10-120 $\mathrm{cm}^{-1}$ is characteristic for shock-induced diamonds [31]. We measured FWHM value for Sadiya as $17.5 \mathrm{~cm}^{-1}$ which reflects the nature of shock metamorphism in the meteorite samples. The intensity ratio of the Raman diamond $\left(\mathrm{I}_{\mathrm{D}}\right)$ and graphite $\left(\mathrm{I}_{\mathrm{G}}\right)$ peaks in graphitic carbon was attributed by Wopenka et al. and ranging for fairly well ordered graphite $\left(\mathrm{I}_{\mathrm{D}} / \mathrm{I}_{\mathrm{G}}<0.5\right)$, disordered graphite $\left(0.51<\mathrm{I}_{\mathrm{D}} / \mathrm{I}_{\mathrm{G}}<1.1\right)$, and glassy carbon $\left(\mathrm{I}_{\mathrm{D}} / \mathrm{I}_{\mathrm{G}}>1.1\right)[22,34]$. We measure the intensity ratio of diamond and graphite peaks of Sadiya and found to be $\sim 0.53$. It implies to the evidence of disordered nature of graphite in Sadiya. The peaks at $1455 \mathrm{~cm}^{-1}$ and $1490 \mathrm{~cm}^{-1}$ are related to phonon modes with $q_{0}$, which are activated by the disorder induced by small grain sizes in nanocrystalline or amorphous diamond [35]. 


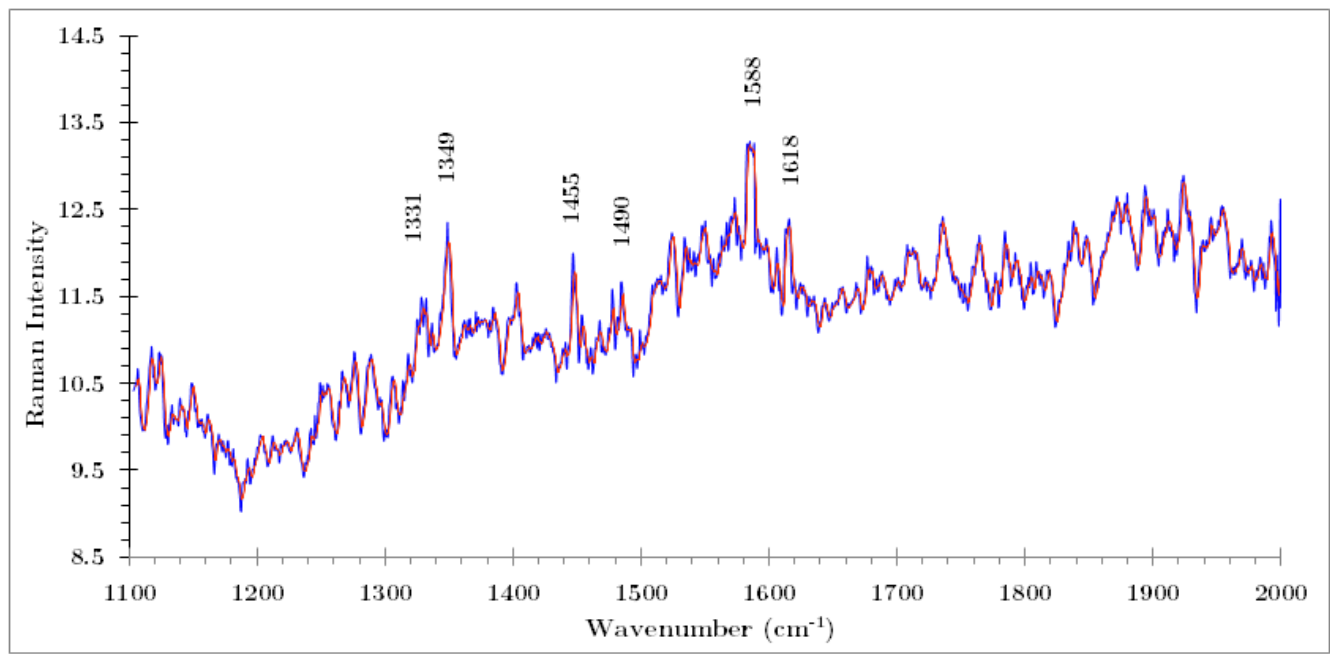

Figure1. Raman spectrum of graphite nodule in Sadiya meteorite sample showing two peaks, at $1349 \mathrm{~cm}^{-1}$ and peak at $\sim 1588-1618 \mathrm{~cm}^{-1}$. The diamond peak appears at $1331 \mathrm{~cm}^{-1}$ (see Appendices).

The infrared spectrum of Sadiya (Fig. 2) reveals numerous absorption bands in 400-1400 $\mathrm{cm}^{-1}$ indicating the presence of silicates. The observed band at $508 \mathrm{~cm}^{-1}$ can be interpreted as $\mathrm{Si}-\mathrm{O}$ and $\mathrm{Mg}-\mathrm{O}$ vibration modes in enstatite $\left(\mathrm{MgSiO}_{3}\right)$ with slight shifts in the matrix [36, 37]. Generally, below 500 $\mathrm{cm}^{-1}$ (e.g. the observed peaks between 465-494 $\mathrm{cm}^{-1}$ ) olivines containing different divalent cations (M$\mathrm{SiO}_{4}$, where $\mathrm{M}$ is $\mathrm{Mg}^{2+}, \mathrm{Fe}^{2+}, \mathrm{Mn}^{2+}$, or $\mathrm{Ca}^{2+}$ ) exhibit a fairly uniform infrared spectrum in which individual bands are sensitive to cation composition [27]. The Si-O asymmetric stretching vibration (TO2-T2O5) is observed in between $974-1057 \mathrm{~cm}^{-1}$. The peaks arise in between $916-974 \mathrm{~cm}^{-1}$ is assigned to the Si-O asymmetric vibration (TO3), whereas the peak arises at $879 \mathrm{~cm}^{-1}$ due to (T2O7-TO4). The appearance of these bands is due to the Si-O mode originating from different silicate (mostly meta- and ortho-silicates) present in the meteorite. The observed bands at $684,722,874,918$, and $1057 \mathrm{~cm}^{-1}$ can be attributed to pyroxene.

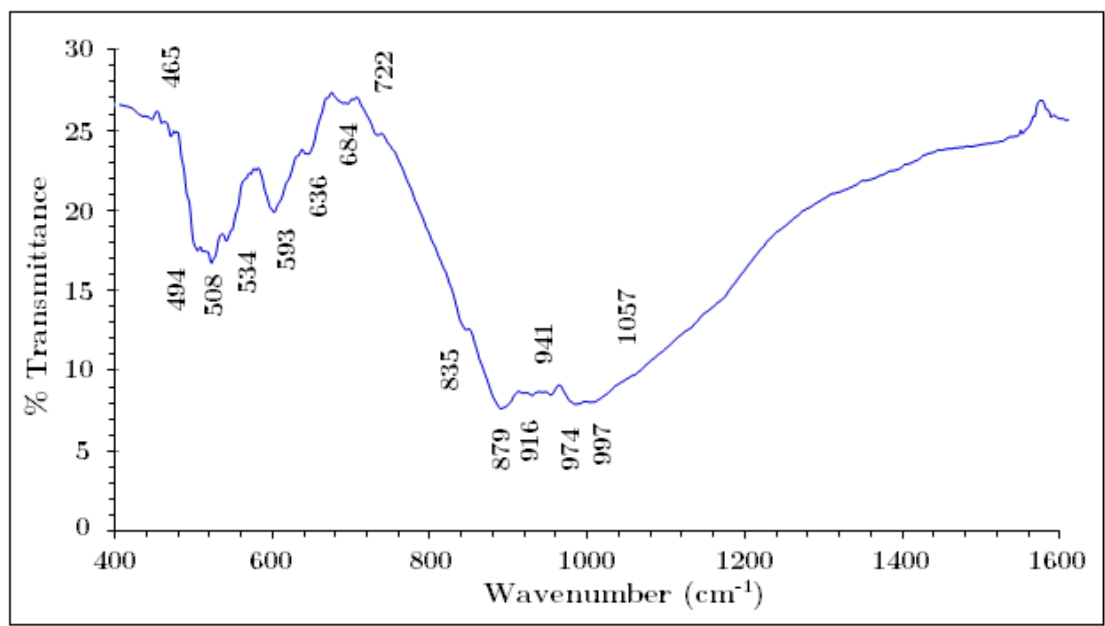

Figure 2. The infrared spectra of Sadiya meteorite in the spectral region $400-1600 \mathrm{~cm}^{-1}$, silicate is recorded in 400 $1000 \mathrm{~cm}^{-1}$ region.

The organic aliphatic C-H stretching features in meteorites have been observed by many authors (e.g. [38-40]). During their studies, three major peaks are found in the 2700-3000 $\mathrm{cm}^{-1}$ region, corresponding to aliphatic hydrocarbon stretching features. The pair of peaks at $2922 \mathrm{~cm}^{-1}$ and $2851 \mathrm{~cm}^{-1}$ corresponds to the asymmetrical and symmetrical stretching vibrations of $\mathrm{CH}_{2}$ in an aliphatic hydrocarbon. The peaks at $2958 \mathrm{~cm}^{-1}$ and $2865 \mathrm{~cm}^{-1}$ correspond to the asymmetrical and symmetrical stretching vibrations 
of $\mathrm{CH}_{3}$ also in an aliphatic hydrocarbon [38-40]. The infrared spectrum of the Sadiya meteorite in the range $2700-3000 \mathrm{~cm}^{-1}$ is shown in Fig. 3. The observed peaks at $2920 \mathrm{~cm}^{-1}$ and $2848 \mathrm{~cm}^{-1}$ correspond to the asymmetrical and symmetrical stretching vibrations of $\mathrm{CH}_{2}$, whereas the peak at $2957 \mathrm{~cm}^{-1}$ arises due to the asymmetrical stretching vibrations of $\mathrm{CH}_{3}[10]$. The $\mathrm{C}-\mathrm{H}$ bending vibrations of aliphatic hydrocarbons are generally observed at $\sim 1466 \mathrm{~cm}^{-1}$ and $\sim 1379 \mathrm{~cm}^{-1}$, but these peaks are not observed in the present analyses. Extraterrestrial organic compounds present in carbonaceous chondrites known to exhibit large structural diversity, which is a feature contrasting with terrestrial biogenic organic compounds [41]. Potiszil et al. have carried out high pressure spectroscopic measurements on organic materials and suggested that the formation of the hydrogen bonds has implications for the origination of macromolecular organic matter $(\mathrm{MOM})$ in the extraterrestrial materials. Such high-pressure-induced hydrogen-bond formation is likely a process by which aromatic MOM precursors could have cross-linked to generate the organic polymers found within extraterrestrial bodies [42].

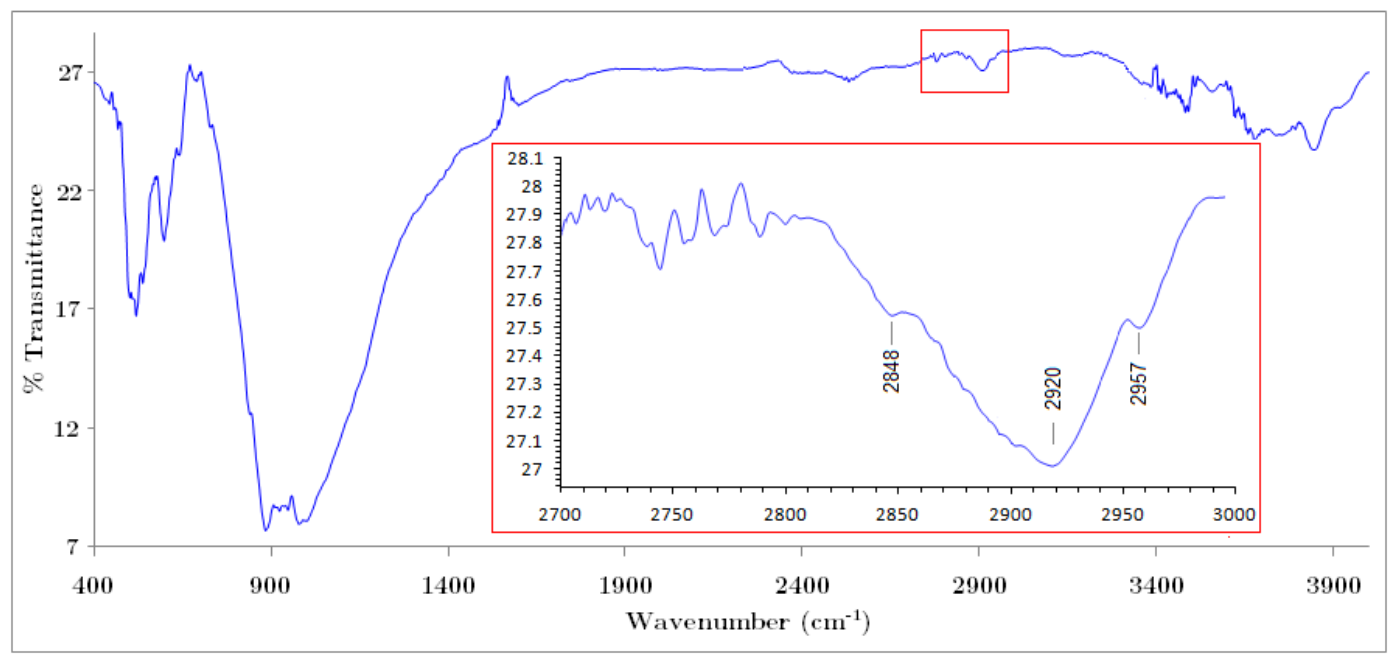

Figure 3.The infrared spectra of Sadiya meteorite in the spectral region $400-4000 \mathrm{~cm}^{-1}$, C-H features is recorded in 2800-3000 $\mathrm{cm}^{-1}$ region (Inset).

\section{Conclusion}

The Raman and infrared spectroscopic results provide the first evidence for the possible existence of organic compounds in Sadiya meteorite. The Raman spectra exhibit the diamond and graphite (D and $\mathrm{G}$ carbon bands) peaks correspondingly observed at $1331 \mathrm{~cm}^{-1}, 1349 \mathrm{~cm}^{-1}$ and $1588-1618 \mathrm{~cm}^{-1}$. The peak intensity of these peaks reflects the degree of ordering. The Raman peak position $1618 \mathrm{~cm}^{-1}$ is the evidence of disordered graphite in the Sadiya meteorite. The peak appears at $1331 \mathrm{~cm}^{-1}$ is accepted for diamond. The presence of $1455 \mathrm{~cm}^{-1}$ in Raman spectra indicates that the formation of nanodiamonds in Sadiya is similar to the chemical vapour deposition process. The full wave at half maximum value 17.5 $\mathrm{cm}^{-1}$ reflects the shock metamorphism in the meteorite samples. The diamond and graphite peaks intensity ratio $(\sim 0.53)$ indicates the disordered nature of graphite in Sadiya. The infrared analysis is consistent to the Raman spectroscopic results. The weak absorption bands found in $2700-3000 \mathrm{~cm}^{-1}$ of Sadiya are indicative to organic compounds. Infrared spectra indicate the presence of aliphatic hydrocarbons in the Sadiya meteorite. Similar organic features have been observed by Lawless et al. and Saikia et al. in $\mathrm{H}$ type ordinary chondrites [10, 43].

Acknowledgments. We are grateful to the anonymous reviewers for their constructive comments, which have improved this manuscript. We thank Directors, National Geophysical Research Institute (NGRI), Hyderabad and Indian Institute of Technology, Guwahati (IITG) for providing analytical facilities for characterization of the meteorite. We thank PLANEX, PRL, and Space Application Centre, Department of Space, Government of India, for their valuable support. We also thank to Dr. S. Sarmah, 
IIT Guwahati, and Dr. J.R. Chetia, Dibrugarh University for their assistance in the spectroscopic analysis.

\section{References}

1. H.Y. McSween Jr., Meteorites and Their Parent Planets, Cambridge, New York, 1999.

2. G. R. Huss, "Ubiquitous interstellar diamond and $\mathrm{SiC}$ in primitive chondrites: Abundance reflect metamorphism", Nature vol.347, pp.159-162, 1990.

3. A. Gucsik, O. Ott, E. Marosits, A. Karczemsk, M. Kozanecki, and M. Szurgot. "Micro-Raman study of nanodiamonds from Allende meteorite". Organic Matter in Space, vol. 251, pp.335-339, 2008.

4. T. L. Daulton, D. D. Eisenhour, T. J. Bernatowitz, R. S. Lewis, and P. R. Buseck, "Genesis of presolar diamonds: Comparative high-resolution transmission electron microscopy study of meteoritic and terrestrial nano-diamonds", Geochimica et Cosmochimica Acta, vol. 60, pp. 4853-4872, 1996.

5. M. Nakamizo, R. Kammereck and P. L. Walker, Jr. "Laser Raman studies on carbons". Carbon, vol. 12, pp. 259$267,1974$.

6. D.G. McCulloch and S. Prawer, "The effect of annealing and implantation temperature on the structure of C ionbeamirradiated glassy carbon". Journal of Applied Physics, vol.78, pp.3040-3047, 1995.

7. R. Escribano, J.J. Sloan, N. Siddique, N. Sze and T. Dudev, "Raman spectroscopy of carbon-containing particles". Vibrational Spectroscopy, vol. 26, pp.179-186, 2001.

8. H.Tao, J. Moser, F. Alzina, Q. Wang and C.M. Sotomayor-Torres, "The morphology of graphene sheets treated in an ozone generator". Journal of Physical Chemistry C, vol.115, pp.18257-18260, 2011.

9. I. Gilmour, "Structural and isotopic analysis of organic matter in carbonaceous chondrites in Meteorites, comets, and planets", Treatise on Geochemistry, vol. 1. Elsevier, 2003, pp. 269-290.

10. B. J. Saikia, G. Parthasarathy and N.C. Sarmah, "Fourier transform infrared spectroscopic characterization of Dergaon H5 chondrite: Evedence of aliphatic organic compound", Nature and Science, vol. 7, pp.45-51.

11. P. Fraundorf, R. I.Patel, P. R. Swan, M. Walker, F. Adar and J.J. Freeman, "Vibrational microspectroscopy of interplanetary dust in the laboratory", in Microbeam analysis, California: San Francisco Press. pp. 188-190, 1982.

12. N. A. Starkey, I. A. Franchi and C. M. O'd. Alexander, "A Raman spectroscopic study of organic matter in interplanetary dust particles and meteorites using multiple wavelength laser excitation", Meteoritics and Planetary Science, vol. 48, pp.1800-1822, 2013.

13. N. A. Starkey and I. A. Franchi, "Insight into the silicate and organic reservoirs of the comet forming region", Geochimica et Cosmochimica Acta, vol.105, pp.73-91, 2013.

14. L. Bonal, E.Quirico, M. Bourot-Denise and G.Montagnac, "Determination of the petrologic type of CV3 chondrites by Raman spectrscopy of included organic matter", Geochimica et Cosmochimica Acta, vol. 70, pp.1849-1863, 2006.

15. L. Bonal, M. Bourot-Denise, E. Quirico, G. Montagnac and E. Lewin. "Organic matter and metamorphic history of CO chondrites". Geochimica et Cosmochimica Acta, vol.71, pp.1605-1623, 2007.

16. H. Busemann, C. M. O. D. Alexander and L. R. Nittler, "Characterization of insoluble organic matter in primitive meteorites by microRaman spectroscopy", Meteoritics and Planetary Science, vol. 37, pp.1387-1416, 2007.

17. E. Dobrica, C. Engrand, E. Quirico, G. Montagnac and J. Duprat, "Raman characterization of carbonaceous matter in CONCORDIA Antarctic micrometeorites", Meteoritics and Planetary Science, vol. 46, pp.1363-1375, 2011.

18. L. J. Allamandola, S. A. Sandford and B.Wopenka, "Interstellar polycyclic aromatic hydrocarbons and the carbon in interplanetary dust particles and meteorites", Science, vol. 237, pp.56-59, 1987.

19. B. Wopenka, "Raman observations on individual interplanetary dust particles", Earth and Planetary Science Letters, vol. 88, pp.221-231, 1988.

20. E. Quirico, J. N. Rouzaud, L. Bonal, and G. Montagnac, "Maturation grade of coals as revealed by Raman spectroscopy: Progress and problems", Spectrochimica Acta A, vol. 61, pp.2368-2377, 2005.

21. A. Rotundi, G. A. Baratta, J. Borg, J. R. Brucato, H. Busemann, L. Colangeli, L. d'Hendecourt, Z. Djouadi, G.Ferrini, I. A. Franchi, M. Fries, F. Grossemy, L.P.Keller, V. Mennella, K. Nakamura, L.R. Nittler, M.E. Palumbo, S.A Sandford., F. J. Steele and B. Wopenka, "Combined micro-Raman, micro-infrared, and filed 
emission scanning electron microscope analyses of Comet 81P/Wild 2 particles collected by Stardust". Meteoritics and Planetary Science, vol.43, pp. 367-397, 2008.

22. B.Wopenka, Y. C. Xu, E. Zinner and S. Amari, "Murchison presolar carbon grains of different density fractions: A Raman spectroscopic perspective", Geochimica et Cosmochimica Acta, vol.106, pp.463-489, 2013.

23. L. J. Allamandola, S. A. Sandford, A. G. G. M. Tielens and T. M.Herbst, "Infrared spectroscopy of dense clouds in the C-H stretch region: Methanol and Diamonds", Astrophysical Journal, vol.399, pp.134-146, 1992.

24. L. J. Allamandola, S. A. Sandford, A. G. G. M. Tielens and T. M.Herbst, "Diamond in dense molecular clouds: A challenge to the standard interstellar medium paradigm", Science, vol.260, pp.64, 1993.

25. B. J. Saikia, G. Parthasarathy, R. R. Borah, R. Borthakur and A.J.D. Sarmah, "Meteorite fall at Sadiya: A Raman spectroscopic classification", Journal of Astrophysics and Aerospace Technology, vol. 5, pp.149, 2017.

26. B. J. Saikia, G. Parthasarathy and R.R. Borah, Nanodiamonds and silicate minerals in ordinary chondrites as determined by micro-Raman spectroscopy, Meteoritics and Planetary Science, vol. 52, pp. 1146-1154, 2017.

27. B. J. Saikia, G. Parthasarathy and N.C. Sarmah, "Spectroscopic characterization of olivine $\left[\left(\mathrm{Fe}, \mathrm{Mg}_{2} \mathrm{SiO}_{4}\right]\right.$ in Mahadevpur H4/5 ordinary chondrite", Journal of American Science, vol. 5, pp.71-78.

28. L. Nasdala, D. C. Smith, R. Kaindl and M. A. Zieman, "EMU Notes Mineralogy 6" in Spectroscopic methods in mineralogy, Eotvos University Press, 2004, pp. 281-343.

29. A. El Goresy, P. Gillet, M. Chen, F. Knstler, G. Graup and V. Stahle, "In situ discovery of shock-induced graphite-diamond phase transition in gneisses from the Ries Crater, Germany", American Mineralogist, vol. 86, pp.611-621, 2001.

30. H. Kagi, I. Tsuchida, M.Wakatsuki, K. Takahashi, N. Kamimura, K. Iuchi, and H. Wada, "Proper understanding of downshifted Raman spectra of natural graphite: Direct estimation of laser-induced rise in sample temperature", Geochimica et Cosmochimica Acta, vol. 58, pp.3527-3530, 1994.

31. M. Miyamoto, "Micro-Raman spectroscopy of diamonds in the Canyon Diablo iron meteorite: Implication for the shock origin", Antarctic Meteorite Research, vol. 11, pp.171-177, 1988.

32. R. S.Lewis, T.Ming, J. F.Wacker, E.Anders and E. Steel, "Interstaller diamonds in meteorites", Nature, vol.326, pp.160-162, 1987.

33. A. C. Ferrari and J. Robertson, "Resonant Raman spectroscopy of disordered, amorphous, and diamondlike carbon", Physical Review B, vol. 64, pp.075414, 2001.

34. S. R.Sharma, G. Parthasarathy, T. R. Ravindran, A. K. Arora, and B. Kumar, "Laser Raman spectroscopic studies on graphites from Dharwar craton: A possible metamorphic grade indicator for the host rocks", Journal of Geological Society of India, vol. 55, pp.413-420, 2000.

35. J. Filik, N.Harvey, N. L.Allan, P. W.May, J. E. P.Dahl, S. Liu and R. M. K. Carlson, "Raman spectroscopy of nanocrystalline dimond: An ab intino approach", Physical Review B, vol.74, pp. 035423-035433, 2006.

36. K. Nakamoto, "Infrared and Raman Spectra of Inorganic and Coordination Compounds", John Wiley \& Sons, 1978.

37. B. J. Saikia, G. Parthasarathy, R. R. Borah, M. Satyanarayanan, R. Borthakur and P. Chetia, "Spectroscopy and mineralogy of a fresh meteorite fall Kamargaon (L6) chondrite", Proceedings of the Indian National Science Academy, vol. 83, pp.941-948, 2017.

38. J. W. Salisbury, L. S.Walter, N.Vergo and D. M. Aria, "Infrared (2.1-25 $\mu \mathrm{m}$ ) spectra of minerals", The Johns Hopkins University, 1992.

39. G. Socrates, "Infrared and Raman characteristic group frequencies", Wiley and Sons, 2001.

40. G. Matrajt, J.Borg, P. I.Raynal, Z. Djouadi, L. d.Hendecourt, G. Flynn, and D. Deboffe, "FTIR and Raman analyses of the Tagish Lake meteorite: Relationship with the aliphatic hydrocarbons observed in the Diffuse Interstellar Medium", Astronomy \& Astrophysics, vol.416, pp.983-990, 2004.

41. Z. Martins, "Organic Chemistry of Carbonaceous Meteorites," Elements, vol. 7, pp. 35-40, 2011.

42. C. Potiszil, W. Montgomery and M. A. Sephton, "Effects of Pressure on Model Compounds of Meteorite Organic Matter", ACS Earth and Space Chemistry, vol.1, pp.475-482, 2017.

43. J. G. Lawless, C. E. Folsome and K. A. Kvenvolden, "Organic matter in meteorites", Scientific American, vol.226, pp.38- 46, 1972. 


\section{Appendix 1:}

Box-whisker plot for wavelength and intencity of the Raman spectra of Sadiya meteorite which is shown in Figure 1.
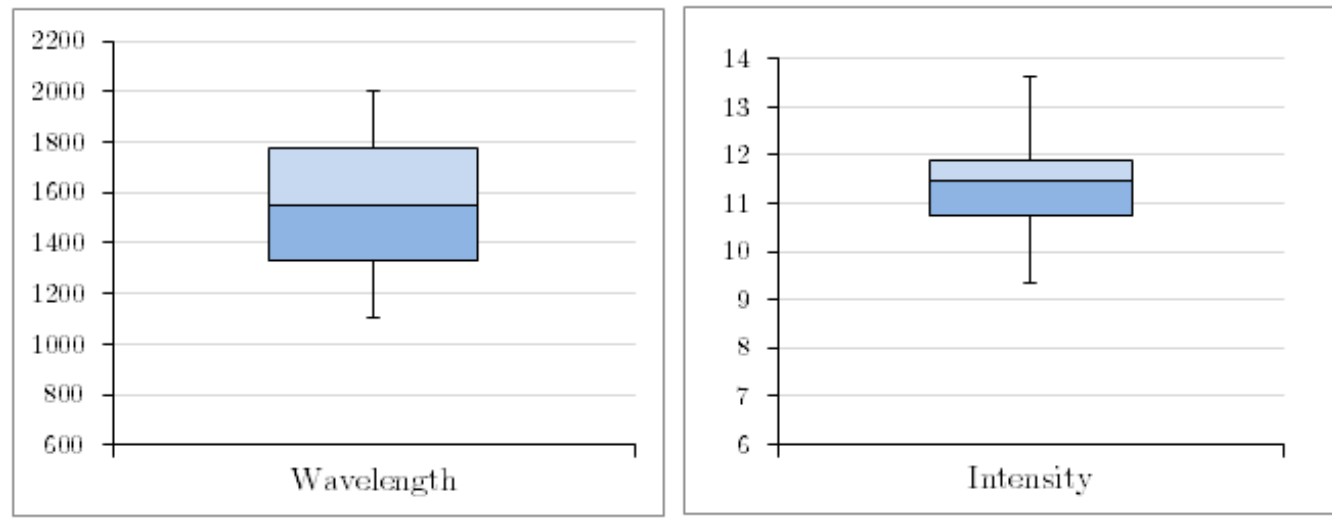

\begin{tabular}{lrr}
\hline & Wavelength & Intensity \\
\hline Min & 1103.505127 & 9.02513657 \\
Q1 & 1327.628815 & 10.7398189 \\
Median & 1552.037109 & 11.4648919 \\
Q3 & 1775.876312 & 11.9008698 \\
Max & 2000.000000 & 13.2854107 \\
\hline Box 1 -Hidden & 1327.628815 & 10.7398189 \\
Box 2 -Lower & 224.4082947 & 0.72507303 \\
Box 3-Upper & 223.8392029 & 0.43597794 \\
\hline Whisker Top & 224.1236877 & 1.3845409 \\
Whisker Bottom & 224.1236877 & 1.7146823 \\
\hline
\end{tabular}

\section{Appendix 2:}

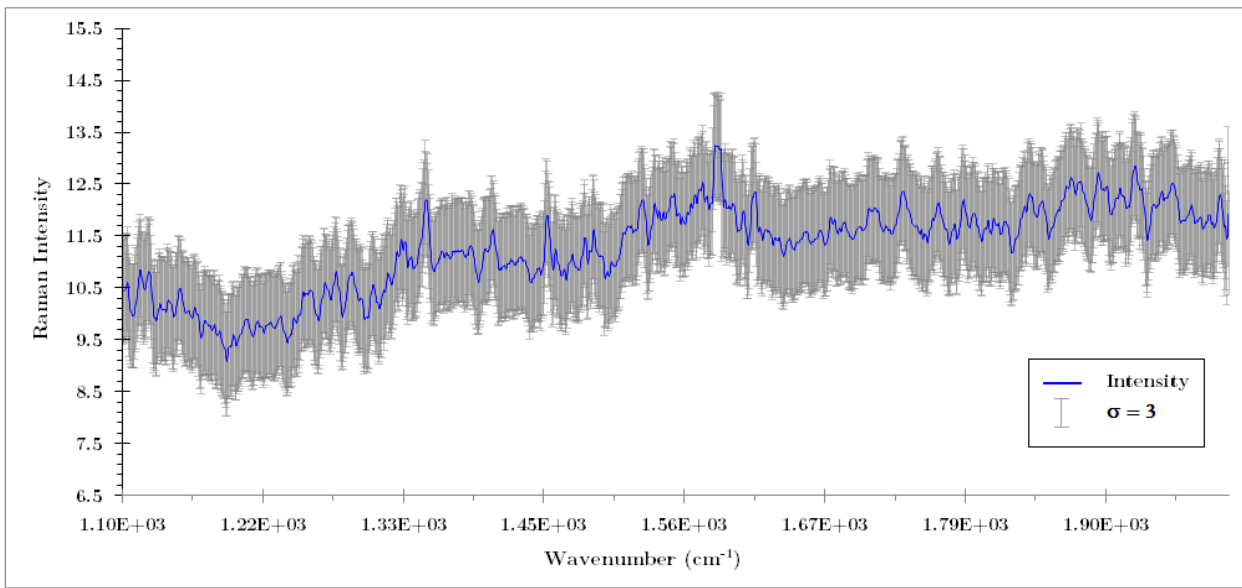

This figure represents the variation of intensity of Raman spectra (Figure 1) at standard deviation $(\sigma=3)$. 